\title{
A Slant Transform Watermarking for Copyright Protection of Satellite Images
}

\author{
Xunzhan Zhu, Anthony T.S. Ho \\ Division of Information Engineering \\ School of Electrical and Electronic Engineering \\ Nanyang Technological University \\ Email: xzzhu@pmail.ntu.edu.sg
}

\begin{abstract}
In this paper, we propose a digital watermarking algorithm based on the Slant transform for the copyright protection of satellite images. Designed to match basis vectors to areas of constant luminance slope, the Slant transform is good for compact energy in "smooth" images. However, our earlier research work suggests that in terms of watermarking, it works better for textured images, for the energy spread becomes a significant advantage in watermarking and offers a good spread of middle to higher frequencies with significant energies for robust information hiding. This has brought us to consider protecting the copyright of digital satellite images using Slant transform watermarking, which is commonly highly textured. An analytical comparative study on the performance of the Slant transform adapting our earlier watermarking schemes for fast Hadamard transform will be performed based on its robustness against various Stirmark attacks.
\end{abstract}

\section{Introduction}

With the tremendous development of the Internet, the online purchasing and distribution of satellite images can now be performed relatively easily. It follows that the copyright protection and authentication of digital satellite images is increasingly becoming more and more important. To counter this growing information security problem of illegal distribution and counterfeiting, a novel watermarking scheme based on fast Hadamard transform for copyright protection of satellite images has been proposed in our previous papers [1,2]. The experiment results suggest that this transform could provide a good "hidden" space for digital watermarking.

In this paper, we propose an improved digital watermarking algorithm based on the Slant transform for the copyright protection of satellite images. The Slant transform has been applied to many image processing applications, such as transform coding and image restoration [3,4,5] along with many fast algorithms have been proposed for it [6]. The Slant transform has many similar properties to the Walsh-Hadamard transform. In terms of transform coding, the Slant transform is considered to be a sub-optimum orthogonal transform for energy compaction. For digital watermarking, the energy spread becomes a significant advantage, as there is now a good spread of middle to higher frequencies with significant energies for robust information hiding. In particular, our earlier-research work suggests the above property of the Slant transform is even more significant when applied to highly textured images [7], which has brought us to consider protecting the copyright of digital satellite images using Slant transform watermarking.

The proposed algorithm applied to satellite images will be evaluated based on its robustness against various Stirmark attacks [8]. The performance results of the Slant transform for satellite image watermarking against Hadamard transform will also be presented.

The rest of this paper is organized as follows. In Section 2 , the Slant transform and its relation to Hadamard transform is briefly reviewed. Then, our proposed watermarking method is elaborated in Section 3. Simulation results are presented in Section 4, these include comparison of the Slant transform to the Hadamard transform in $[1,2]$. Finally, the conclusions are given in Section 5 .

\section{The Slant Transform}

Let $[U]$ be the original image of size $N \times N$, its 2D-Slant transform is given by [5]

$$
[V]=\left[S_{n}\right][U]\left[S_{n}\right]^{T}
$$

where $\left[S_{n}\right]$ is the $N \times N$ unitary Slant matrix. The inverse transformation to recover $[U]$ from the transform components $[V]$ is given by

$$
[U]=\left[S_{n}\right]^{T}[V]\left[S_{n}\right]
$$

The Slant transform is a member of the orthogonal transforms. It has a constant function for the first row, and has a second row which is a linear (slant) function of the column index.

The Slant transform matrix of order two is given by 


$$
S_{2}=\frac{1}{2^{1 / 2}}\left[\begin{array}{cc}
1 & 1 \\
1 & -1
\end{array}\right]
$$

The Slant matrix of order four is obtained by the operation

$$
S_{4}=\frac{1}{2^{1 / 2}}\left[\begin{array}{ccccc}
1 & 0 & \vdots & 1 & 0 \\
a_{4} & b_{4} & \vdots & -a_{4} & b_{4} \\
\cdots & \cdots & \cdots & \cdots & \cdots \\
0 & 1 & \vdots & 0 & -1 \\
-b_{4} & a_{4} & \vdots & b_{4} & a_{4}
\end{array}\right]\left[\begin{array}{ccc}
S_{2} & \vdots & 0 \\
& \vdots & \\
\cdots & \cdots & \cdots \\
& \vdots & \\
0 & \vdots & S_{2}
\end{array}\right]
$$

where $a_{4}$ and $b_{4}$ are scaling constants. The orthonormality conditions lead to

$$
a_{4}=2 b_{4} \text { and } b_{4}=\frac{1}{5^{1 / 2}}
$$

Equation (4) can be generalized to give the Slant matrix of order $N$ in terms of the Slant matrix of order $N / 2$ by the recursive relation as given by

$$
\begin{aligned}
& S_{N}=\frac{1}{2^{1 / 2}}\left[\begin{array}{ccccccccc}
1 & 0 & \vdots & & \vdots & 1 & 0 & \vdots & \\
a_{N} & b_{N} & \vdots & 0 & \vdots & -a_{N} & b_{N} & \vdots & 0 \\
\cdots & \cdots & \cdots & \cdots & \cdots & \cdots & \cdots & \cdots & \cdots \\
& 0 & \vdots & I_{(w / 2)-2} & \vdots & & 0 & \vdots & I_{(n / 2)-2} \\
\cdots & \cdots & \cdots & \cdots & \cdots & \cdots & \cdots & \cdots & \cdots \\
0 & 1 & \vdots & & \vdots & 0 & -1 & \vdots & \\
-b_{N} & a_{N} & \vdots & 0 & \vdots & b_{N} & a_{N} & \vdots & 0 \\
\cdots & \cdots & \cdots & \cdots & \cdots & \cdots & \cdots & \cdots & \cdots \\
& 0 & \vdots & I_{(n / 2)-2} & \vdots & & 0 & \vdots & -I_{(n / 2)-2}
\end{array}\right] \\
& \times\left[\begin{array}{ccc} 
& \vdots & \\
S_{N / 2} & \vdots & 0 \\
& \vdots & \\
& \vdots & \\
\cdots & \vdots & \cdots \\
& \vdots & \\
& \vdots & \\
0 & \vdots & S_{N / 2} \\
& \vdots &
\end{array}\right]
\end{aligned}
$$

The matrix $I_{(n / 2)-2}$ is the identity matrix of dimension $(N / 2)-2$. The constants $a_{N}, b_{N}$ may be computed by the formulas

$$
a_{2 N}=\left(\frac{3 N^{2}}{4 N^{2}-1}\right)^{1 / 2}, b_{2 N}=\left(\frac{N^{2}-1}{4 N^{2}-1}\right)^{1 / 2}
$$

In [6], Wang proved that the Slant transform may be approached by a series of steps that gradually change the transform from a Hadamard transform, which demonstrates the close relationship between the Slant transform and the Walsh-Hadamard transform. From our previous study [7], the Slant transform and the Hadamard transform display similar properties when applied to digital watermarking.

\section{Watermarking in Slant Transform Domain}

A generic block diagram of the blind watermarking system [10] is shown in Figure 1. The original image is not necessary at the watermark recovery stage. This refers to a "blind" watermarking process. A visually recognizable pattern is embedded by modifying the Slant transform coefficients of relevant sub-blocks of the host image. The detailed Image-embedded watermark insertion and extraction algorithm are discussed in this section.

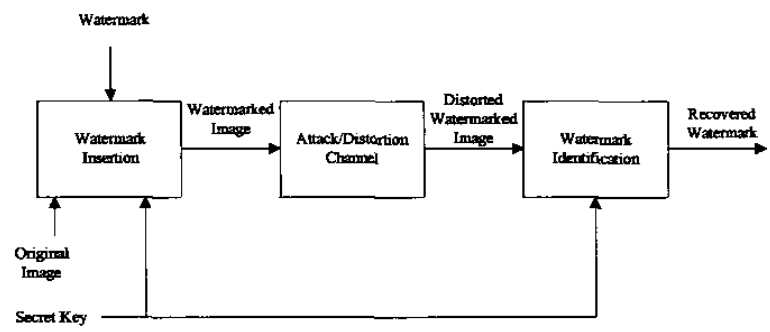

Figure 1 Generic block diagram of a blind watermarking system

Copyright information in the form of a trademark or logo can be created as a pattern for watermarking. In our experiment, a grayscale image of size $64 \times 64$ is used as the watermark. The watermark insertion process is shown in Figure 2. We adapt a similar image-embedded watermark insertion algorithm as in [1], while using the Slant transform domain instead of the Hadamard transform domain. The algorithm can be described as follows.

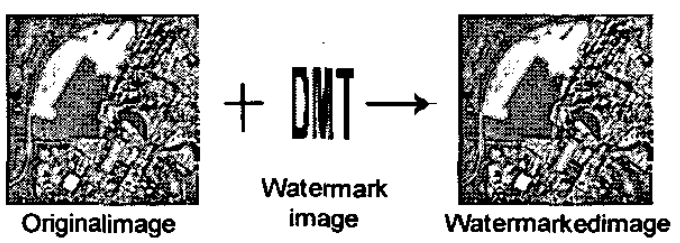

Figure 2 Image-embedded watermarking insertion process

The watermark image, $W(x, y)$, is first transformed into a set of Slant transform coefficients by equation (1). A Slant transform matrix of order 64 is applied to this image, and then a $64 \times 64$ Slant transform coefficients matrix is obtained. The DC component is stored in the secret key file and the AC components are used for watermark embedding.

Let the original image be $I(x, y)$. Similar to the algorithm used in [1,2], it is decomposed into a set of non-overlapped $8 \times 8$ sub-blocks. An m-sequence random number generator is used to select a certain number of sub-blocks for watermark embedding, whose initial seed is also kept in the secret key file. In every selected sub-block, sixteen middle and high frequency coefficients are used for later modulation. The way of the coefficients selection affects the performance of the watermarking scheme significantly. 
The high frequency components are relatively vulnerable to compression operations, while the low frequency components must be retained for visual quality of the watermarked image. Therefore, most existing watermarking schemes choose to embed the watermark into the middle frequency band. In our scheme, embedding locations as shown in Figure 3 are adopted, which are observed, through our experiments, to provide a best tradeoff between robustness and data integrity.

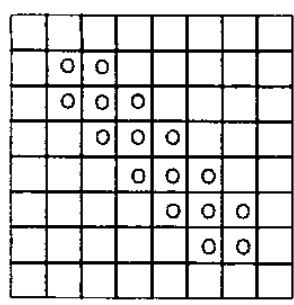

Figure 3 Embedding locations

Let the watermark Slant transform coefficients denoted by $m_{i}$. The AC coefficients of Slant transformed original image sub-blocks, before and after inserting watermark are denoted by $x_{i}$ and $x_{i}^{*}$, respectively, and $i \in(0, n]$, where $n$ is the number of the watermarked coefficients to be inserted into every sub-block, which is set to 16 in our experiment. The embedding formula is given as follows

$$
x_{i}^{*}=\alpha m_{i}
$$

where $\alpha$ is the watermark strength factor that controls the tradeoff between visual quality of the watermarked image and robustness of the watermarking scheme.

After embedding, the original coefficient $x_{i}$ is replaced by $x_{i}^{*}$ and a new $8 \times 8$ matrix of Slant transform coefficients of image sub-block is obtained. The inverse Slant transform is then applied to the $8 \times 8$ matrix using equation (2) to obtain the luminance matrix of the watermarked image subblock. After performing the watermark insertion for all the selected sub-blocks of the original image, a watermarked image, $I^{\prime}(x, y)$, is obtained. At the same time, as indicated earlier, the secret key file has been generated for subsequent decoding.

In watermark detection, the positions of the sub-blocks with watermark embedded are computed using the seed of the $\mathrm{m}$-sequence and initial state number that is stored in the key file. All the selected sub-blocks are Slant transformed. Let these coefficients denoted by $x_{i}^{*}$ and the retrieved watermark Slant transform coefficients by $m_{i}^{\prime}, i \in(0, n]$, where $n$ is the number of the watermarked coefficients to be inserted in every sub-block. The watermark extraction formula is given by

$$
m_{i}^{\prime}=\frac{x_{i}^{*}}{\alpha}
$$

The extracted AC coefficients and the DC component stored in the key file are rearranged into a $64 \times 64$ Slant transform coefficients matrix. The extracted watermark image, $W^{*}(x, y)$, is then obtained by an inverse Slant transform using equation (2).

\section{Simulation Results}

We use two $512 \times 512$ gray-scale satellite images with distinct texture to test our algorithm. The original and watermarked images are shown in Figure 4. Results show that there are no perceptually visible degradations on the watermarked images with a PSNR of $37.43 \mathrm{~dB}$ for Singapore and $40.65 \mathrm{~dB}$ for Dolomites.

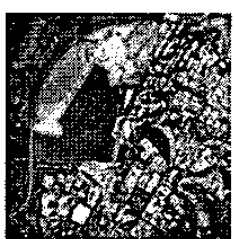

(a)
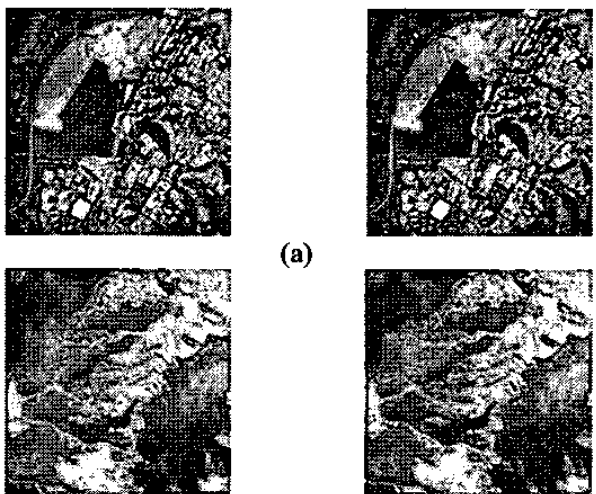

(b)

Figure 4 Original images (left) and watermarked images (right):

\begin{tabular}{|c|c|c|}
\hline Image operations & Extracted watermark & Correlation \\
\hline $\begin{array}{c}\text { JPEG } \\
\text { compression of } \\
\text { factor } 80\end{array}$ & & 0.8858 \\
\hline $\begin{array}{c}\text { JPEG } \\
\text { compression of } \\
\text { factor } 50\end{array}$ & Wha & 0.7432 \\
\hline $\begin{array}{l}\text { White additive } \\
\text { noise of } 1.5 \%\end{array}$ & a) & 0.8173 \\
\hline Scaling 2.00 & What & 0.7441 \\
\hline Sharpening & an & 0.7930 \\
\hline
\end{tabular}
(a) Singapore; (b) Dolomites.

Table 1 Results of some Stirmark tests for Singapore 
The Slant transform watermarking scheme is benchmarked using Stirmark 4.0 [8]. Sample results of the Singapore are shown in Table 1. We can see that the Slant transform watermarking is robust to noise and common image processing techniques.

We also compare the robustness of the Slant scheme to the Hadamard scheme in $[1,2]$ against the typical JPEG compression attack. The results are shown in Figures 5 . We can see that, for the two typical satellite images, the Slant transform survive JPEG compression far better than the Hadamard transform. Since the Slant transform is designed to match basis vectors to areas of constant luminance slope, it is good for compact energy in "smooth" images. However, in terms of watermarking, it works better for "textured" images, for the energy spread becomes a significant advantage in watermarking and offers a good spread of middle to higher frequencies with significant energies for robust information hiding. It follow therefore that the Slant transform is very robust when applied for watermarking satellite images, which are commonly highly textured.

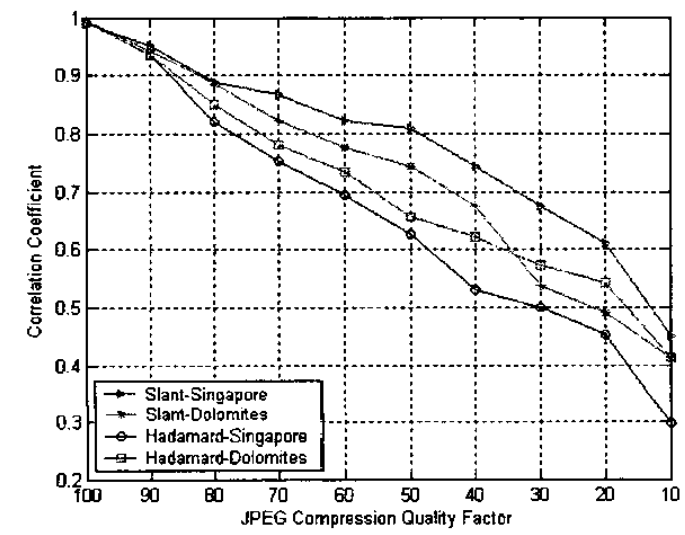

Figure 5 Performance comparisons between Slant transform and Hadamard transform, using two images Singapore and Dolomites.

\section{Conclusion}

This paper has presented a robust watermarking technique for embedding grayscale image watermark into a satellite image based on the Slant transform. The embedding and extracting processes have been described in details. The Slant transform has more useful middle and high frequency bands than several high gain transforms, especially for highly textured satellite images, which provides a good "hidden" space for digital watermarking. It also offers a significant advantage in shorter processing time and ease of hardware implementation than commonly used DCT and DWT techniques. This research is based on our previous work on the Hadamard transform [1,2]. Comparison results between the Slant transform and the Hadamard transform have also been presented.

\section{References}

[1] Ho, A.T.S., J. Shen, S. H. Tan, "Digital image-in image watermarking technique for copyright protection of satellite images using the fast Hadamard transform," IGARSS02', June 24-16, 2002, Toronto, Canada.

[2] Ho, A.T.S., J. Shen, S. H. Tan, "A robust digital imagein-image watermarking technique for satellite images," ICICS'01, 15-18 October, 2001, Singapore.

[3] Clarke, R. J., Transform Coding of Images, New York: Academic Press, 1985

[4] W. K. Pratt, L. R. Welch, and W. H. Chen, "Slant transform for image coding," Proc. Symp. Appl. Walsh Function, March 1972.

[5] W. K. Pratt, W.H. Chen, and L.R. Welch, "Slant transform coding," IEEE Trans. Communications, vol. COM-22, No. 8, Aug. 1974.

[6] Z. D. Wang, "New algorithm for the slant transform," IEEE trans. Pattern Analysis and Machine Intelligence, vol. pami-4, pp. 551-555, Dec. 1982.

[7] Ho, A.T.S., X. Zhu, J. Shen, "Slant transform watermarking for digital images," Visual Communications and Image Processing (VCIP'03), SPIE, Accepted for session on Image and Video Security and Watermarking I, 8-11, July 2003, Lugano, Switzerland.

[8] Available at: http://www.cl.cam.ac.uk/ fapp2/watermarking/stirmark.

[9] I. J. Cox, J. Kilian, F. T. Leighton, and T. Shamoon, "Secure spread spectrum watermarking for multimedia," IEEE Trans. Image Processing, vol. 6, pp. 1673-1687, Dec. 1997.

[10] S. Katzenbeisser and A.P.F. Petitcolas, Information Hiding Techniques for Steganography and Digital Watermarking, Artech House, Boston, 2000. 